\title{
Monetary Services Aggregation under Uncertainty: A Behavioral Economics Extension Using Choquet Expectation
}

\author{
William A. Barnett \\ University of Kansas, Lawrence, and Center for Financial Stability, NY City \\ williamabarnett@gmail.com \\ Qing Han \\ University of Kansas, Lawrence \\ qinghan@ku.edu \\ Jianbo Zhang \\ University of Kansas, Lawrence \\ jbzhang@ku.edu
}

August 1, 2018

\begin{abstract}
A central tenet of behavioral economics is that the axioms producing expected utility maximization by consumers are too strong to be descriptive of rational behavior. The existing theory of monetary services aggregation under risk assume expected utility maximization. We extend those results to uncertainty under weaker axiomatic assumptions by using Choquet expectations. Choquet integration reduces to Riemann integration as a special case under the stronger assumption of additive probability measure, not accepted in the literature on behavioral economics. Our theoretical results on monetary services aggregation are generalizations of prior results, nested as special cases of our results under stronger behavioral assumptions.
\end{abstract}

JEL Classification: E41, G12, C43

Keywords: Uncertainty Aversion, User Cost, Choquet Expectation, Monetary Aggregation

Note: Address correspondence to Qing Han, Economics Department, University of Kansas, 1460 Jayhawk Blvd., Snow Hall, Lawrence, KS 66045-7585; email: qinghan@ku.edu. 


\section{Introduction}

Expected utility maximization is based on behavioral assumptions having strong normative support, as in Bayesian econometrics. But as a descriptive model of rational behavior by consumers, the growing field of behavioral economics widely rejects expected utility maximization. ${ }^{1}$ We consider monetary aggregation theory under non-expected utility and derive the model implications for monetary asset user costs and optimal portfolio selection, when agents are uncertainty averse. Specifically, under non-expected utility maximization, we permit consumers' subjective probability distributions under uncertainty to be inconsistent with linear probabilistic additivity. In contrast most economists assume that subjective probabilities must sum to one, as a normative representation of how rational consumers "should behave," although that assumption is inconsistent with findings in the behavioral economics literature about how consumers actually behave.

We use the term uncertainty, as opposed to risk, since we do not assume that the objective probability distribution is known to the consumer. To be consistent with the relevant literature, we also assume that the utility function, in a dynamical context, exhibits a recursive structure: current period utility depends on expected future utility as well as on current consumption. Under the assumption that this recursive dependence is separable, the non-expected utility we use is expected utility under a nonadditive probability measure. The resulting model aims to separate the more subtle "uncertainties" from quantifiable "risk." The objective of this paper is to unravel the implications of monetary aggregation theory, when consumers' behavior deviates from expected utility maximization.

The literature on monetary services aggregation derived from aggregation theory began with Barnett $(1978,1980)$. The resulting literature separates the investment motive from the services motive, when monetary assets yield interest as well as providing monetary services. If the interest rates paid to monetary asset

\footnotetext{
11 The central role in behavioral economics of non-expected utility maximization has been emphasized by Kahneman and Tversky (1979), Tversky and Kahneman (1992), and Thaler (1993). Also see Grabisch (1996) and Chateauneuf and Cohen (2010), among many others.
} 
holders were a monetary service, then coal mines, real estate, and the entire capital stock of the country would be money.

That literature was subsequently extended to include risk by Poterba and Rotemberg (1987) and Barnett (1995), when current period interest is not known until the end of the period. Barnett, Liu, and Jensen (1997) further extended to the case of CCAPM (consumption capital asset pricing) and subsequently by Barnett and $\mathrm{Wu}$ (2005), who extended to the case of intertemporal nonseparability providing larger risk adjustments than produced by CCAPM. Recently, Keating and Smith (2018) explore the usefulness of aggregation theoretic monetary aggregates in Taylor rules policies in the framework of a rational expectations model. ${ }^{2}$

We permit the possibility that consumers' behavior might deviate from expected utility maximization. Monetary assets are durable goods and thus have user cost prices. Deriving those user costs is a fundamental step in producing monetary aggregates from economic aggregation theory. As a result, our research begins by determining the implications of non-expected utility for the user costs of monetary assets.

The expected utility of von Neumann and Morgenstern received axiomatic support from Savage (1954), using a prior subjective probability that sums up to one. The Savage axioms and the resulting expected utility maximization imputed to consumers have been building blocks of many economic models. Yet Allais (1953) and the Ellsberg (1961) paradox find that human being's behavior frequently falls outside the prediction of expected utility. One group of models, seeking to generalize expected utility theory, distinguishes between risk and uncertainty, as defined by Knight (1921) and further developed by Bewley (2002). In that literature, risk exists when economic agents know the objective probabilities, which do sum to one. Under uncertainty, the objective probabilities are not known to economic agents, and the resulting behavior of economic agents need not be representable by a subjective probability distribution, having the same measure theoretic properties of

\footnotetext{
2 Other important contributions to this literature include Barnett (2012), Barnett and Chauvet (2011), Barnett, Keating, and Kelly (2011), Belongia and Chalfant (1989), Barnett and Ireland (2014; 2015a,b;2016), Serletis and Gogas (2014), and Serletis and Rahman (2013).
} 
the unknown objective probability distribution. Although the subjective joint probability of the union of all possible outcomes is necessarily one, the sum of the probabilities of each of those independent, separate outcomes is not necessarily one. $^{3}$

We follow that approach. In particular, the model we use is built on a nonadditive probability measure. This approach has its foundations in Schmeidler's findings: if probabilities reflect people's willingness to bet, those probabilities need not be additive. An axiomatic treatment of nonadditive probability models can be found in Schmeidler (1986, 1989), Gilboa (1987, 2009), and Gilboa and Schmeidler (1989). While this approach does not capture all of the objections to expected utility maximization in the behavioral economics literature, this generalization does permit a formal mathematical solution producing an elegant generalization to expected utility maximization under weaker assumptions than the Savage axioms and is consistent with Tversky and Kahneman's (1992) approach.

We find that nonadditive probability measure yields boundaries to the user cost of monetary assets, depending on whether the marginal utility and rate of return are comonotonic or countermonotonic. This does not mean, however, that user costs under nonadditive expectation are only subject to inequality constraints. If there exists an underlying probability measure to properly define the nonadditive probabilities, we find that the user cost has a rank-dependent expected utility representation. This solution has an expected utility form, but uses transformed distorted additive probabilities as weights. The rank dependence is much less restrictive than might appear to be the case, since there is always a permutation to line up the objective function in an ascending/descending order.

We also find that under optimality there is a user cost interval within which the agent will not hold any position in the monetary asset. When the user cost is below the lower limit of this interval, she will want to buy more of the monetary asset. When the user cost is above the upper limit of this interval, she will want to sell the

\footnotetext{
${ }^{3}$ For a formal definition of nonadditive probability, see the first paragraph of section 2.1 below.
} 
monetary asset (short). The two limits of this interval constitute the reserve prices for transactions, if the agent's belief reflects uncertainty aversion. This result does not hinge on her attitude towards risk. Our model thus is capable of explaining why there are situations under which people are not active in changing their monetary asset portfolios. A reasonable individual may not behave consistently with Savage's model. Maximizing utility under a nonadditive prior can provide a useful rationale for observed behavior in the market. When probabilities become additive, the model reduces to von Neumann-Morgenstern expected utility case. The existing publications on monetary aggregation under risk become special cases of our analysis and hence are formally nested within our theory.

The rest of the paper is organized as follows. In section 2 we introduce the model and the associated nonadditive probability measure, solve for the user cost under uncertainty aversion, and derive the user cost boundaries. In section 3 we find the conditions under which the user cost has a rank-dependent expected utility representation. In section 4 we consider the consumer's problem from an asset pricing perspective and demonstrate our main theorem providing the user cost interval within which no trade will happen. In section 5 we conclude the paper. The appendix contains the mathematical proofs of theorems and useful lemmas.

\section{The Model}

\subsection{Utility Function and Uncertainty Averse}

When we say the probability is nonadditive, we mean that if $A$ and $B$ are two disjoint events in the sample space $\Omega$, such that $A \cup B=\Omega$, with their probabilities being $v(A)$ and $v(B)$ respectively, then $v(A)+v(B) \neq 1$, although $v(A \cup B)=1$. As explained below, uncertainty aversion will imply $v(A)+v(B)<1$. Under a nonadditive probability measure, the proper way to define an integral is no 
longer Riemann but Choquet. Under these conditions, Riemann integration suffers from discontinuity, nonmonotonicity, and ambiguity (dependence upon the form of the utility function). Suppose there is a function $f \geq 0$. Then the Choquet (1954) integral integrates over rectangles horizontally:

$$
\int f d v=\int_{0}^{\infty} v(s \mid f(s) \geq t) d t,
$$

where the right hand side is a standard Riemann integral. ${ }^{4}$ The Choquet integral has many attractive properties, such as reflecting linear translations multiplied by a positive coefficient. But generally it is not additive, unless the functions under evaluation are comonotonic, a property that will be relevant to some of our results below.

Under uncertainty, the utility function under our consideration is in the form of nonexpected utility as follows:

$$
V_{t}=U\left(c_{t}, \mathbf{m}_{t}, E_{t}^{C} V_{t+1}\right)=u\left(c_{t}, \mathbf{m}_{t}\right)+\beta \int V_{t+1} d v,
$$

where $c_{t}$ is the date $t$ consumption of goods, $\mathbf{m}_{t}$ is the vector of monetary assets, and $E_{t}^{C} V_{t+1}$ is expected future utility, conditional on all information at time t. ${ }^{5}$ We use a superscript $C$ on the expectation operator to denote Choquet expectation. In this uncertainty context, $U(\cdot)$ is the aggregator function through which current consumption, all monetary assets, and expected future utility are aggregated. We follow canonical macroeconomic models to allow time separability, where $\beta$ is the subjective discount factor and $V_{t+1}$ is tomorrow's utility in each of tomorrow's states. Without the separability assumption, the discount factor would be the derivative of $U(\cdot)$ with respect to its third argument.

We further assume there exists a linearly homogenous aggregator function, $M_{t}=M\left(\mathbf{m}_{t}\right)$, such that:

\footnotetext{
${ }^{4}$ For a more formal, but conveniently accessible, definition and discussion of Choquet integration, see its Wikipedia entry at https://en.wikipedia.org/wiki/Choquet_integral.

5 Distinguishing attitudes towards risk from behavior towards intertemporal substitution is beyond the scope of this paper. Once we include monetary assets in the utility function, the effects of Epstein and Zin (1989) or Weil's (1990) generalized isoelastic utility are much harder to find. But it could be a topic worth pursuing.
} 


$$
u\left(c_{t}, \mathbf{m}_{t}\right)=F\left[c_{t}, M\left(\mathbf{m}_{t}\right)\right] .
$$

In this paper, additive probability is denoted by $P$, while capacity (nonadditive probability or "charge") is denoted by $v$, so that $\int(\cdot) d v$ is the Choquet integral.

More formally, suppose that $S$ is a finite set of states of nature, and in every period there are a finite number of $n$ different states. Let $\mathcal{F}$ be the $\sigma$-algebra generated by the events on $S$. Then capacity $v$ on a measurable space $(S, \mathcal{F})$ is a real-valued set function $v: \mathcal{F} \rightarrow[0,1]$, such that $v(\phi)=0, v(\mathrm{~S})=1$, and $v(S) \leq v(B)$ for all $\mathrm{A} \subseteq \mathrm{B} \in \mathcal{F}$. An example of capacity could be $v=P^{\alpha}$. In this case, $\alpha$ measures the agent's attitude towards uncertainty. If $\alpha=1$, then capacity reduces to an additive prior probability measure. As this result illustrates, nonadditive probability reflects both the presence of uncertainty and the agent's attitude towards it.

Using the example from the beginning of this section, $v(A)+v(B)<1=v(A \cup B)$ is equivalent to concluding that the agent's decisions reflect uncertainty aversion. ${ }^{6}$ Schmeidler $(1986,1989)$ defines uncertainty aversion in terms of probability capacity by

$$
v(A)+v(B) \leq v(A \cup B)+v(A \cap B),
$$

although that definition is not universally accepted. That condition is also known as supermodularity, convexity, or 2-monotonicity of $v$.

The states of nature are a natural partition of the sample space S. If today's nature is denoted by $s$, we denote the state of nature tomorrow by $s^{\prime}$. With a somewhat informal notation for $V$, it can be useful to rewrite the utility function (1) in terms of states for any given sequence, $\mathbf{X}=\left\{c_{t}, \mathbf{m}_{t}\right\}_{t=0}^{T}$, as $V(\mathbf{X})=\lim _{T \rightarrow \infty}\left\{u\left(c_{0}, \mathbf{m}_{0}\right)+\beta \int\left[u\left(c_{1}, \mathbf{m}_{1}\right)+\cdots+\beta \int u\left(c_{T}, \mathbf{m}_{T}\right) v_{s_{T-1}}\left(d s_{T}\right) \cdots\right] v_{s}\left(d s_{1}\right)\right\}$.

This facilitates the calculation of Choquet integral using Riemann integrals.

\footnotetext{
${ }^{6}$ We avoid use of the word "ambiguity," which is usually defined to mean that the agent vaguely perceives the probability of a particular state in a range. This possibility is outside the scope of this paper.
} 


\subsection{Equilibrium}

The agent holds two types of assets, monetary assets and nonmonetary assets. Nonmonetary assets provide only investment return, while monetary assets provide both investment return and monetary service flows, which we seek to measure. The budget constraints are

$$
\begin{gathered}
W_{t}=p_{t} c_{t}+\sum_{i=1}^{L} p_{t} m_{i t}+\sum_{j=1}^{K} p_{t} k_{j t}, \\
W_{t+1}=\sum_{i=1}^{L} R_{i, t+1} p_{t} m_{i t}+\sum_{j=1}^{K} \tilde{R}_{j, t+1} p_{t} k_{j t}+y_{t+1},
\end{gathered}
$$

where $W_{t}$ is the agent's wealth in period t, $p_{t}$ is the true cost of living index, $c_{t}$ is consumption of goods, and $y_{t+1}$ is income from all other sources, received at the beginning of $t+1$. The variables, $m_{i t}$ and $k_{j t}$, denote the quantities of monetary asset $i$ and nonmonetary asset $j$ respectively. The interest rate $R_{i, t+1}$ is the gross rate of return on monetary asset $m_{i t}$, while the interest rate $\tilde{R}_{j, t+1}$ is the gross return of nonmonetary asset $k_{j t}$.

Suppose $L$ and $K$ are the number of the two types of assets in the agent's portfolio. Since nonmonetary assets do not provide service flows, other than their investment rates of return, it follows that $\tilde{R}$ is higher than $R$. Combining equation (5) and (6) yields the following flow of funds equation:

$$
p_{t} c_{t}=\sum_{i=1}^{L}\left[R_{i t} p_{t-1} m_{i, t-1}-p_{t} m_{i t}\right]+\sum_{j=1}^{K}\left[\tilde{R}_{j t} p_{t-1} k_{j, t-1}-p_{t} k_{j t}\right]+y_{t} .
$$

Hence, the individual's consumption of goods is funded each period from the proceeds from rolling over the monetary assets and nonmonetary assets and from all other income. Note that equation (7) is the one used in Barnett (1980) and Barnett, Liu, and Jensen (1997) to facilitate comparison of our results with the existing literature. 
The agent maximizes lifetime discounted utility (4), subjects to the flow of funds constraint (7). The resulting Bellman equation is:

$$
\begin{aligned}
& V_{s}\left(W_{t}\right)=\sup _{\left\{c_{t}, \mathbf{m}_{t}, \mathbf{k}_{t}\right\}}\left\{u\left(c_{t}, \mathbf{m}_{t}\right)+\beta \int V_{s^{\prime}}\left(W_{t+1}\right) v_{s}\left(d s^{\prime}\right)\right\} \\
& \text { s.t. } \quad p_{t} c_{t}=\sum_{i=1}^{L}\left[R_{i t} p_{t-1} m_{i, t-1}-p_{t} m_{i t}\right]+\sum_{j=1}^{K}\left[\tilde{R}_{j t} p_{t-1} k_{j, t-1}-p_{t} k_{j t}\right]+y_{t} .
\end{aligned}
$$

Here $V_{s}\left(W_{t}\right)$ denotes the Bellman value function. The agent is also subject to the following transversality condition:

$$
\lim _{t \rightarrow \infty} \beta^{t} \frac{\partial V^{*}}{\partial W_{t}^{*}} W_{t}^{*}=0,
$$

with $*$ denoting the solution value from the optimization.

After substituting from the Benveniste-Scheinkman equation, the first order conditions (Euler equations) with respect to consumption become

$$
\frac{\partial u}{\partial c_{t}}=\beta E_{t}^{C}\left[\frac{\partial u}{\partial c_{t+1}} \tilde{R}_{j, t+1} \frac{p_{t}}{p_{t+1}}\right],
$$

while the first order conditions with respect to monetary assets become

$$
\frac{\partial u}{\partial m_{i t}}=\frac{\partial u}{\partial c_{t}}-\beta E_{t}^{C}\left[\frac{\partial u}{\partial c_{t+1}} R_{i, t+1} \frac{p_{t}}{p_{t+1}}\right]
$$

The contemporaneous real user-cost price of the services of monetary asset $i$ is the marginal rate of substitution between the monetary asset and consumption,

$$
\pi_{i t}=\frac{\frac{\partial u}{\partial m_{i t}}}{\frac{\partial u}{\partial c_{t}}}=\frac{\frac{\partial u}{\partial c_{t}}-\beta E_{t}^{C}\left[\frac{\partial u}{\partial c_{t+1}} R_{i, t+1} \frac{p_{t}}{p_{t+1}}\right]}{\beta E_{t}^{C}\left[\frac{\partial u}{\partial c_{t+1}} \tilde{R}_{j, t+1} \frac{p_{t}}{p_{t+1}}\right]}=\frac{\frac{\partial u}{\partial c_{t}}-\beta E_{t}^{C}\left[\frac{\partial u}{\partial c_{t+1}} r_{i, t+1}\right]}{\beta E_{t}^{C}\left[\frac{\partial u}{\partial c_{t+1}} \tilde{r}_{j, t+1}\right]} .
$$

For notational convenience, we convert the nominal gross returns, $R_{i, t+1}$ and $\tilde{R}_{j, t+1}$, to the corresponding real gross rates of return, $r_{i, t+1}=R_{i, t+1} \frac{p_{t}}{p_{t+1}}$ and $\tilde{r}_{j, t+1}=\tilde{R}_{j, t+1} \frac{p_{t}}{p_{t+1}}$. Since the expectation $E_{t}^{C}(\cdot)$ is not additive, it is the Choquet integral. Also note that under the weak separability condition (2), we have 


$$
\frac{\partial u}{\partial m_{i t}}=\frac{\partial F}{\partial M_{t}} \frac{\partial M_{t}}{\partial m_{i t}}
$$

Substituting the definition of the user cost, we acquire:

$$
\frac{\partial M_{t}}{\partial m_{i t}}=\pi_{i t} \frac{\frac{\partial u}{\partial c_{t}}}{\frac{\partial F}{\partial M_{t}}}
$$

Taking the total differential of the monetary aggregator function, $M_{t}=M\left(\mathbf{m}_{t}\right)$, yields

$$
d M_{t}=\sum_{i=1}^{L} \frac{\partial M_{t}}{\partial m_{i t}} d m_{i t}=\frac{\frac{\partial u}{\partial c_{t}}}{\frac{\partial F}{\partial M_{t}}} \sum_{i=1}^{L} \pi_{i t} d m_{i t}=\frac{\frac{\partial u}{\partial c_{t}}}{\frac{\partial F}{\partial M_{t}}} \sum_{i=1}^{L} \pi_{i t} m_{i t} d \log m_{i t}
$$

Since $M\left(\mathbf{m}_{t}\right)$ is linearly homogenous of degree one, Euler's theorem simplifies (13) to

$$
M_{t}=\sum_{i=1}^{L} \frac{\partial M_{t}}{\partial m_{i t}} m_{i t}=\frac{\frac{\partial u}{\partial c_{t}}}{\frac{\partial F}{\partial M_{t}}} \sum_{i=1}^{L} \pi_{i t} m_{i t}
$$

Dividing equation (13) by (14) yields the Divisia index

$$
d \log M_{t}=\sum_{i=1}^{L} s_{i t} d \log m_{i t},
$$

where $s_{i t}=\frac{\pi_{i t} m_{i t}}{\sum_{l=1}^{L} \pi_{l t} m_{l t}}$ is the user cost valued expenditure share. We conclude that the resulting Divisia quantity index is in exactly the same form as in Barnett (1980), with the only difference being that the user costs now are computed under a nonadditive probability measure.

\subsection{User-Cost Boundaries}


We now return to the user-cost, $\pi_{i t}$, in equation (12). Because the expectation is non-additive, we no longer have $E_{t}\left[\frac{\partial u}{\partial c_{t+1}} r_{i, t+1}\right]=\operatorname{Cov}\left(\frac{\partial u}{\partial c_{t+1}}, r_{i, t+1}\right)+E_{t}\left(\frac{\partial u}{\partial c_{t+1}}\right) E_{t}\left(r_{i, t+1}\right) . \quad$ Instead, we have the following theorem:

Theorem 1. If $\frac{\partial u}{\partial c_{t+1}}, r_{i, t+1} \geq 0$ are comonotonic, then

$$
E_{t}^{C}\left[\frac{\partial u}{\partial c_{t+1}} r_{i, t+1}\right] \geq E_{t}^{C}\left(\frac{\partial u}{\partial c_{t+1}}\right) E_{t}^{C}\left(r_{i, t+1}\right)
$$

If $v$ is submodular, while $\frac{\partial u}{\partial c_{t+1}}$ and $r_{i, t+1}$ are countermonotonic, then:

$$
E_{t}^{C}\left[\frac{\partial u}{\partial c_{t+1}} r_{i, t+1}\right] \leq E_{t}^{C}\left(\frac{\partial u}{\partial c_{t+1}}\right) E_{t}^{C}\left(r_{i, t+1}\right)
$$

The proof of this theorem is in the appendix.

Comonotonicity is defined as follows. For every pair of states, $\mathrm{s}_{1}^{\prime}, \mathrm{s}_{2}^{\prime} \in \mathrm{S}$,

$$
\left[\frac{\partial u}{\partial c_{t+1}}\left(s_{1}^{\prime}\right)-\frac{\partial u}{\partial c_{t+1}}\left(s_{2}{ }^{\prime}\right)\right]\left[r_{i, t+1}\left(s_{1}^{\prime}\right)-r_{i, t+1}\left(s_{2}^{\prime}\right)\right] \geq 0 .
$$

Then the marginal utility and the rate of return increase or decrease at the same time. Countermonotonicity reverses the direction of the above inequality. Hence, under nonadditive probabilities, we do not have covariances, but we have inequalities. Equation (17) corresponds to uncertainty loving which is unusual and not the focus of this paper. ${ }^{7}$

Barnett, Liu, and Jensen (1997) proved, in their Theorem 1, that the user cost of the services of monetary assets under risk aversion has an additional adjustment term not appearing in the risk free user cost. That adjustment term is about

\footnotetext{
7 Although we do not consider uncertainty loving to be relevant monetary aggregation theory, there are circumstances under which it is not unreasonable. If we think about gain-loss asymmetry, when people particularly hate to lose what they have already had, such an extreme loss aversion might lead people to behave in an uncertainty loving way in the domain of losses.
} 
covariances, as in all CCAPM risk adjustments. The Barnett, Liu, and Jensen's risk adjusted user cost is a special case of our result. If the probability measure is additive, so that uncertainty is removed, risk aversion is all that is left. Then the Choquet expectation in equation (12) becomes the linearly additive expectation, and covariances appear.

When the agent is not only risk averse but also uncertainty averse, then equation (12) cannot be further simplified by collecting covariances. We end up with inequalities giving rise to boundaries on user costs. In the next section, we will see that equality solutions do exist for $E_{t}^{C}\left[\frac{\partial u}{\partial c_{t+1}} r_{i, t+1}\right]$, but those again hold as special cases of Choquet expectation. Our case nests Barnett, Liu and Jensen's (1997) result. If we further assume away both uncertainty aversion and risk aversion, we will have the perfect certainty case. Then equation (12) reduces to the user cost derived in Barnett (1980).

It is convenient to work on rates of returns, $r_{i, t+1}$, which are usually assumed to be stationary, so that taking averages is meaningful. But marginal utility, $\partial u / \partial c_{t+1}$, is not observed and difficult to estimate. Therefore we reinterpret equation (12) in terms of a stochastic discount factor, which, although still not observable, is much easier to estimate. We assume the agent has not passed the bliss point, so that $\partial u / \partial c_{t} \geq 0$. Given date $t$ information, uncertainty at time $t$ has been resolved, and $\partial u / \partial c_{t}$ can be treated as a constant. By the positive homogeneity of Choquet integral, equation (12) can be written as

$$
\pi_{i t}=\frac{1-E_{t}^{C}\left[\beta \frac{\partial u / \partial c_{t+1}}{\partial u / \partial c_{t}} r_{i, t+1}\right]}{E_{t}^{C}\left[\beta \frac{\partial u / \partial c_{t+1}}{\partial u / \partial c_{t}} \tilde{r}_{j, t+1}\right]}=\frac{1-E_{t}^{C}\left[Q_{t+1} r_{i, t+1}\right]}{E_{t}^{C}\left[Q_{t+1} \tilde{r}_{j, t+1}\right]},
$$

where we denote by $Q_{t+1}=\beta \frac{\partial u / \partial c_{t+1}}{\partial u / \partial c_{t}}$ the pricing kernel. Note that from equation (10) and (11), we have respectively 


$$
\begin{gathered}
1=E_{t}^{C}\left[Q_{t+1} \tilde{r}_{j, t+1}\right], \\
\pi_{i t}=1-E_{t}^{C}\left[Q_{t+1} r_{i, t+1}\right] .
\end{gathered}
$$

Based on equation (19), a reinterpretation of Theorem 1 is that, if $Q_{t+1}, r_{i, t+1} \geq 0$ and $Q_{t+1}, \tilde{r}_{j, t+1} \geq 0$ are both comonotonic, then:

$$
\pi_{i t} \leq \frac{1-E_{t}^{C}\left(Q_{t+1}\right) E_{t}^{C}\left(r_{i, t+1}\right)}{E_{t}^{C}\left(Q_{t+1}\right) E_{t}^{C}\left(\tilde{r}_{j, t+1}\right)}
$$

If $v$ is submodular and $Q_{t+1}$ and the rate of return on both the monetary and non-monetary assets are countermonotonic, we have:

$$
\pi_{i t} \geq \frac{1-E_{t}^{C}\left(Q_{t+1}\right) E_{t}^{C}\left(r_{i, t+1}\right)}{E_{t}^{C}\left(Q_{t+1}\right) E_{t}^{C}\left(\tilde{r}_{j, t+1}\right)} .
$$

Since returns tend to move together, the dual satisfaction of comonotonic (or countermonotonic) with $Q_{t+1}$ is not restrictive.

Therefore when the probability measure is nonadditive, Choquet expectation produces boundaries to the user cost of monetary assets. More specifically, assume the real rates of return on both types of assets are positive and the substitution effect on intertemporal consumption dominates, so that the comonotonicity between $Q_{t+1}$ and $r_{i, t+1}\left(\tilde{r}_{j, t+1}\right)$ is satisfied. Then the calculated user cost should be lower than $\frac{1-E_{t}^{C}\left(Q_{t+1}\right) E_{t}^{C}\left(r_{i, t+1}\right)}{E_{t}^{C}\left(Q_{t+1}\right) E_{t}^{C}\left(\tilde{r}_{j, t+1}\right)}$. On the other hand, if the agent were uncertainty loving and the income effect dominates the intertemporal allocation of consumption, the countermonotonicity between $Q_{t+1}$ and $r_{i, t+1}\left(\tilde{r}_{j, t+1}\right)$ is satisfied. Then any calculated user cost would be incorrect, if it were lower than $\frac{1-E_{t}^{C}\left(Q_{t+1}\right) E_{t}^{C}\left(r_{i, t+1}\right)}{E_{t}^{C}\left(Q_{t+1}\right) E_{t}^{C}\left(\tilde{r}_{j, t+1}\right)}$.

\section{Rank-Dependent Representation}


The existence of derived boundaries is not our only result under nonadditive probabilities. In this section we show that under some circumstances, there exists a linear solution for equation (21). Suppose $\mathbf{P}=\left(P_{1}, P_{2}, \cdots, P_{n}\right)^{T}$ is an additive probability vector satisfying $\sum_{s=1}^{n} P_{s}=1$, and suppose there is a probability measure $\mu$ such that for some nondecreasing function $f:[0,1] \rightarrow[0,1]$ with $f(0)=0$ and $f(1)=1$, the capacity $v=f(\mu)$ is well-defined. Then a new, additive, probability vector $\mathbf{P}^{\uparrow}$ is permissible to order events as follows:

$$
\begin{aligned}
& \mathbf{P}^{\uparrow}=\left(P_{1}^{\uparrow}, P_{2}^{\uparrow}, \cdots, P_{n}^{\uparrow}\right)^{T} \\
& =\left[1-f\left(\sum_{s \geq 2} P_{s}\right), f\left(\sum_{s \geq 2} P_{s}\right)-f\left(\sum_{s \geq 3} P_{s}\right), \cdots, f\left(\sum_{s \geq n-1} P_{s}\right)-f\left(P_{n}\right), f\left(P_{n}\right)\right]^{T} .
\end{aligned}
$$

If the agent is uncertainty averse, $f(\cdot)$ should be concave, so higher states are weighted less. Such a transformed probability could be tailored for accumulative lottery outcomes, where $x_{1} \leq \cdots \leq x_{n}$ in a lottery $\left(x_{1}, P_{1} ; \cdots ; x_{n}, P_{n}\right)$. This observation is a reason we choose the notation $\uparrow$ on the left side of equation (24). Take the distorted probability as an example, in which $f(\mu)=\mu^{\alpha}$, where $\alpha \in(0,+\infty)$, and $\mu$ is the probability measure relative to which the additive probabilities $P_{s}$ are given. Then $P_{t}^{\uparrow}=\left(\sum_{s=t}^{n} P_{s}\right)^{\alpha}-\left(\sum_{s=t+1}^{n} P_{s}\right)^{\alpha}$, so that the above probability vector $\mathbf{P}^{\uparrow}$ becomes

$$
\mathbf{P}^{\uparrow}=\left[1-\left(\sum_{s \geq 2} P_{s}\right)^{\alpha},\left(\sum_{s \geq 2} P_{s}\right)^{\alpha}-\left(\sum_{s \geq 3} P_{s}\right)^{\alpha}, \cdots,\left(\sum_{s \geq n-1} P_{s}\right)^{\alpha}-\left(P_{n}\right)^{\alpha},\left(P_{n}\right)^{\alpha}\right]^{T} .
$$

The higher states are weighted less in this example when, $\alpha>1$.

Similarly, we define another probability vector, $\mathbf{P}^{\downarrow}$, for decumulative outcomes $x_{1} \geq \cdots \geq x_{n}$ as follows:

$$
\begin{aligned}
& \mathbf{P}^{\downarrow}=\left(P_{1}^{\downarrow}, P_{2}^{\downarrow}, \cdots, P_{n}^{\downarrow}\right)^{T} \\
& =\left[f\left(P_{1}\right), f\left(\sum_{s \leq 2} P_{s}\right)-f\left(P_{1}\right), \cdots, f\left(\sum_{s \leq n-1} P_{s}\right)-f\left(\sum_{s \leq n-2} P_{s}\right), 1-f\left(\sum_{s \leq n-1} P_{s}\right)\right]^{T} .
\end{aligned}
$$


If the agent is uncertainty averse, higher states are weighted more. This approach is also the method proposed by Yaari (1987) to deal with the violation of continuity and monotonicity in Kahneman and Tversky's (1979) prospect theory.

We therefore have the following lemma showing that Choquet expectation has an expected utility solution, but with a transformed probability measure on ordered utilities.

Lemma 2. Suppose $P$ is an additive probability measure for any capacity $v=f(\mu)$ that is well supported by the probability measure $\mu$ and for any nonnegative function $u \in \mathbb{R}_{+}^{n}$. Then the Choquet integral has a rank-dependent expected utility representation:

$$
\begin{aligned}
& \int u_{s} v(d s)=\mathbf{u}^{T} \mathbf{P}^{\uparrow}=\sum_{s=1}^{n} u_{s} P_{s}^{\uparrow}, \text { if } u \text { is weakly increasing in } s, \\
& \int u_{s} v(d s)=\mathbf{u}^{T} \mathbf{P}^{\downarrow}=\sum_{s=1}^{n} u_{s} P_{s}^{\downarrow}, \text { if } u \text { is weakly decreasing in } s,
\end{aligned}
$$

where $\mathbf{P}^{\uparrow}$ and $\mathbf{P}^{\downarrow}$ are state-reweighted probability vectors defined above.

The proof of the lemma is in the appendix. With this result, if $Q_{t+1} r_{i t+1}$ is weakly increasing in $s^{\prime}$, as can always be done by permutation, we have

$$
\pi_{i t}=1-E_{t}^{C}\left[Q_{t+1} r_{i, t+1}\right]=1-\sum_{s^{\prime}=1}^{n} Q_{t+1} r_{i, t+1} P_{s^{\prime}}^{\uparrow}
$$

If $Q_{t+1} r_{i, t+1}$ is weakly decreasing in $s^{\prime}$, then

$$
\pi_{i t}=1-E_{t}^{C}\left[Q_{t+1} r_{i, t+1}\right]=1-\sum_{s^{\prime}=1}^{n} Q_{t+1} r_{i, t+1} P_{s^{\prime}}^{\downarrow}
$$

Therefore, in addition to deriving inequality bounds, we also have an alternative solution. Choquet expectation relative to $v$ coincides with an expected utility model defined by $f(\cdot)$. This expected utility requires rank dependence, so that the product 
$Q_{t+1}\left(s^{\prime}\right) r_{i t+1}\left(s^{\prime}\right)$ must be either weakly increasing or weakly decreasing in $s^{\prime}$. The correspondence between Choquet expectation and the rank-dependent representation does not always exist. Rather, the rank-dependent expected utilities are a special case of Choquet expected utility, a case in which the underlying probability measure $\mu$ exists and contains sufficient information to define $v$.

The Ellsberg paradox is a violation of this condition and therefore has no rank-dependence representation. In those cases, there does not exist an underlying measure, providing all we need to know about events. Potentially, Choquet expectation is more general, in that it allows us to work on scenarios during which our capabilities of defining probabilities are limited.

Note that equation (20) also features a similar rank-dependent solution:

$$
\begin{aligned}
& \sum_{s^{\prime}=1}^{n} Q_{t+1} \tilde{r}_{j, t+1} P_{s^{\prime}}^{\uparrow}=1 \text { if } Q_{t+1} \tilde{r}_{j, t+1} \text { is weakly increasing in } s^{\prime}, \\
& \sum_{s^{\prime}=1}^{n} Q_{t+1} \tilde{r}_{j, t+1} P_{s^{\prime}}^{\downarrow}=1 \text { if } Q_{t+1} \tilde{r}_{j, t+1} \text { is weakly decreasing in } s^{\prime} .
\end{aligned}
$$

These two equations provide a useful guidance for estimating the stochastic discount factor, when uncertainty aversion is involved. We can compare equation (20) with the classical asset pricing theory under additive priors. In that case, returns should follow

$$
1=E_{t}\left[Q_{t+1} \tilde{r}_{t+1}\right]
$$

Then one dollar paid today is weighted against how many dollars or units of consumption the agent will get in return tomorrow. If the decision also involves attitudes towards uncertainty, we now see that (32) becomes $1=E_{t}^{C}\left[Q_{t+1} \tilde{r}_{t+1}\right]$.

With the implication of Lemma 2, it becomes clear that even if people manage to evaluate this true equation, as in (30) and (31), the result would still be a special case of our more general theory.

\section{Choice under Uncertainty Aversion: A No Trade Interval}


In this section we reverse the perspective by looking for the portfolio no trade region. Given that $v$ is a probability measure, the value of expected discounted real rate of return exhibits linearity and translation invariance; that is, $E_{t}^{C}\left[\alpha Q_{t+1} r_{i, t+1}+\beta\right]=\alpha E_{t}^{C}\left[Q_{t+1} r_{i, t+1}\right]+\beta$, if $\alpha \geq 0, \beta \in \mathbb{R}$. But this property does not hold, when $\alpha$ is negative. Therefore, we consider $-E_{t}^{C}\left[-Q_{t+1} r_{i, t+1}\right]$ instead, giving rise to the following lemma:

Lemma 3. If the agent is uncertainty averse, the Choquet expected value satisfies

$$
-E_{t}^{C}\left[-Q_{t+1} r_{i, t+1}\right]>E_{t}^{C}\left[Q_{t+1} r_{i, t+1}\right] \text {. }
$$

The proof is in the appendix. Intuitively, adding a constant to a random variable or multiplying a random variable by a positive number will linearly shift the Choquet expectation. This relationship does not hold for negative multipliers. The nonadditivity of the probability causes an asymmetric effect, producing an interval within which there are no transactions of monetary asset $i$. There will be a range of discounted returns from $E_{t}^{C}\left[Q_{t+1} r_{i, t+1}\right]$ to $-E_{t}^{C}\left[-Q_{t+1} r_{i, t+1}\right]$, within which the agent neither want to buy nor to sell the monetary asset. If the discounted return $E_{t}^{C}\left[Q_{t+1} r_{i, t+1}\right]$ is larger than 1 , she will want to buy the monetary asset. If the discounted return $-E_{t}^{C}\left[-Q_{t+1} r_{i, t+1}\right]$ is lower than 1 , she will want to sell this monetary asset (short).

To prove this result, we assume the utility function, $u \geq 0$, is twice continuously differentiable with $u^{\prime}>0$ and $u^{\prime \prime}<0$. We use Jensen's inequality to prove this result. But first we need to verify whether Jensen's inequalities hold under a nonadditive probability measure.

Lemma 4. Let $(S, F, V)$ be a nonadditive probability space, $S^{\prime} \in S$, and let 
$v \in V$ be capacity. Suppose $x_{t+1}\left(s^{\prime}\right)$ is Choquet integrable. If $u$ is a concave function on $[0,+\infty)$, then Jensen's inequality follows:

$$
u\left\{E_{t}^{C}\left[x_{t+1}\left(s^{\prime}\right)\right]\right\} \geq E_{t}^{C}\left\{u\left[x_{t+1}\left(s^{\prime}\right)\right]\right\}
$$

Proof: We produce a second order Taylor expansion of $u\left(x_{t+1}\left(s^{\prime}\right)\right)$ around $E_{t}^{C}\left(x_{t+1}\left(s^{\prime}\right)\right):$

$$
u(x)=u\left[E^{C}(x)\right]+u^{\prime}\left[E^{C}(x)\right]\left[x-E^{C}(x)\right]+\frac{1}{2} u "(\xi)\left[x-E^{C}(x)\right]^{2} .
$$

Taking Choquet expectation on both sides, we have:

$$
\begin{aligned}
E^{C}[u(x)] & =E^{C}\left\{u\left[E^{C}(x)\right]+u^{\prime}\left[E^{C}(x)\right]\left[x-E^{C}(x)\right]+\frac{1}{2} u "(\xi)\left[x-E^{C}(x)\right]^{2}\right\} \\
& =u\left[E^{C}(x)\right]+E^{C}\left\{u^{\prime}\left[E^{C}(x)\right]\left[x-E^{C}(x)\right]+\frac{1}{2} u^{\prime \prime}(\xi)\left[x-E^{C}(x)\right]^{2}\right\} \\
& \leq u\left[E^{C}(x)\right]+E^{C}\left\{u^{\prime}\left[E^{C}(x)\right]\left[x-E^{C}(x)\right]\right\} \\
& =u\left[E^{C}(x)\right]+u^{\prime}\left[E^{C}(x)\right] E^{C}\left[x-E^{C}(x)\right] \\
& =u\left[E^{C}(x)\right]+u^{\prime}\left[E^{C}(x)\right]\left[E^{C}(x)-E^{C}(x)\|v\|\right] \\
& =u\left[E^{C}(x)\right]
\end{aligned}
$$

where $\xi=\lambda x_{t+1}\left(s^{\prime}\right)+(1-\lambda) E_{t}^{C}\left[x_{t+1}\left(s^{\prime}\right)\right]$ with $\lambda \in[0,1]$. The convex case can be proved similarly.

The second line holds because of comonotonic additivity. The constant is comonotonic with any variable. The third line holds because the Choquet integral is monotone and $u^{\prime \prime}(\xi)<0,\left[x-E^{C}(x)\right]^{2} \geq 0$. The fourth line is true because of positive homogeneity, and $u^{\prime}\left[E^{C}(x)\right]$ is a constant. The rest holds because of translation invariance.

So Jensen's inequalities are still satisfied under nonadditive probabilities, and they are satisfied in multivariate case. We now have the main result, as follows:

Theorem 5. Consider a risk neutral or risk averse agent with wealth, $W_{t}$, who is 
considering investing $m_{i t}$ in a monetary asset, yielding a real rate of return $r_{i, t+1}$. Suppose the two conditions in Lemma 4 are satisfied. Denoting the Choquet expected discounted rate of return by $E_{t}^{C}\left[Q_{t+1} r_{i, t+1}\right]$, she will buy this monetary asset, if $1<E_{t}^{C}\left[Q_{t+1} r_{i, t+1}\right]$, or equivalently if $\pi_{i t}<0$. She will sell the asset (short), if $1>-E_{t}^{C}\left[-Q_{t+1} r_{i, t+1}\right]$, or equivalently $\pi_{i t}>-E_{t}^{C}\left[-Q_{t+1} r_{i, t+1}\right]-E_{t}^{C}\left[Q_{t+1} r_{i, t+1}\right]$.

We only sketch the proof. Suppose the agent spends $m_{i t}$ on this monetary asset. Then by Jensen's inequality

$$
E_{t}^{C}\left\{u\left[W_{t}-m_{i t}+m_{i t} \cdot Q_{t+1} r_{i, t+1}\right]\right\} \leq u\left\{E_{t}^{C}\left[W_{t}-m_{i t}+m_{i t} \cdot Q_{t+1} r_{i, t+1}\right]\right\} \leq u\left(W_{t}\right) .
$$

The last inequality holds, if $E_{t}^{C}\left[Q_{t+1} r_{i, t+1}\right] \leq 1$. Therefore the individual is at least as well off not buying anything as holding a positive position in monetary asset $i$. Analogous arguments give rise to selling the asset, if $1>-E_{t}^{C}\left[-Q_{t+1} r_{i, t+1}\right]$. In this circumstance,

$$
\begin{aligned}
& 1-\left[-E_{t}^{C}\left[-Q_{t+1} r_{i, t+1}\right]\right] \\
& =1-E_{t}^{C}\left[Q_{t+1} r_{i, t+1}\right]-\left[-E_{t}^{C}\left[-Q_{t+1} r_{i, t+1}\right]\right]+E_{t}^{C}\left[Q_{t+1} r_{i, t+1}\right]>0 .
\end{aligned}
$$

Since $\pi_{i t}=1-E_{t}^{C}\left[Q_{t+1} r_{i, t+1}\right]$, this condition is equal to $\pi_{i t}>-E_{t}^{C}\left[-Q_{t+1} r_{i, t+1}\right]-E_{t}^{C}\left[Q_{t+1} r_{i, t+1}\right]$, and by Lemma 3 this difference is positive.

Hence $\left[0,-E_{t}^{C}\left[-Q_{t+1} r_{i, t+1}\right]-E_{t}^{C}\left[Q_{t+1} r_{i, t+1}\right]\right]$ is a range of user costs with no trade under uncertainty aversion. If the user cost, $\pi_{i t}$, is lower than zero, we conclude $1<E_{t}^{C}\left[Q_{t+1} r_{i, t+1}\right]$, so the return tomorrow is larger than the one dollar spent on the asset today, and she will buy it. If $\pi_{i t}$ is larger than $-E_{t}^{C}\left[-Q_{t+1} r_{i, t+1}\right]-E_{t}^{C}\left[Q_{t+1} r_{i, t+1}\right]$, then $1>-E_{t}^{C}\left[-Q_{t+1} r_{i t+1}\right]$ and the uncertainty premium is not enough to compensate for the cost of holding the monetary asset. 
She will want to sell it. This range of user costs depends only on the beliefs and attitude towards uncertainty, not on the attitude towards risk.

\section{Concluding Remarks}

In this paper we consider monetary services aggregation theory under uncertainty, as distinguished by Knight (1921) from risk. The agent's attitude towards uncertainty is represented by a probability measure that need not be additive, in accordance with Schmeidler (1986, 1989), Gilboa (1987, 2009), and Gilboa and Schmeidler (1989). We acquire three primary conclusions. First, different from CCAPM risk adjusted user costs incorporating covariances and subject to the "equity premium puzzle" critique, we find that the uncertainty adjusted user cost, in its most general form, produces boundaries. The previously derived perfect certainty user cost and the risk adjusted user cost are special cases of ours, if the probability measure becomes additive. Second, we are able to derive an expected utility analogous solution, using transformed additive probabilities. The resulting model of expectations is a special case of Choquet expectation. Third, user costs under uncertainty produce an interval within which no transactions of monetary assets will occur. This effect is brought about solely by uncertainty aversion, not by risk aversion captured by the utility function. The resulting behavioral corner solutions are not produced or explained by conventional expected utility maximization solutions for monetary asset service user costs.

While our approach does not resolve all of the objections to expected utility maximization in the behavioral economics literature, the approach using Choquet expectations has established connections with the method proposed by Yaari (1987) to deal with the violation of continuity and monotonicity discussed in Kahneman and Tversky's (1979), and is consistent with the use of Choquet integration in Tversky and Kahneman (1992).

\section{Appendix}




\section{Proof of Theorem 1:}

Let $\frac{\partial u}{\partial c_{t+1}}$ and $r_{i, t+1}$ be comonotonic in the sense that for each pair of states, $s_{1}{ }^{\prime}, s_{2}{ }^{\prime} \in S$,

$$
\left[\frac{\partial u}{\partial c_{t+1}}\left(s_{1}^{\prime}\right)-\frac{\partial u}{\partial c_{t+1}}\left(s_{2}^{\prime}\right)\right]\left[r_{i, t+1}\left(s_{1}^{\prime}\right)-r_{i, t+1}\left(s_{2}^{\prime}\right)\right] \geq 0
$$

Suppose $E_{t}^{C}\left[\frac{\partial u}{\partial c_{t+1}} r_{i, t+1}\right]<\infty$ and both $\frac{\partial u}{\partial c_{t+1}}, r_{i, t+1} \geq 0$. For any given $s_{0}$ ', we have by comonotonicity

$$
\left[\frac{\partial u}{\partial c_{t+1}}-\frac{\partial u}{\partial c_{t+1}}\left(s_{0}^{\prime}\right)\right]\left[r_{i t+1}-r_{i, t+1}\left(s_{0}^{\prime}\right)\right] \geq 0
$$

That is

$$
\frac{\partial u}{\partial c_{t+1}} r_{i, t+1}+\frac{\partial u}{\partial c_{t+1}}\left(s_{0}^{\prime}\right) r_{i, t+1}\left(s_{0}^{\prime}\right) \geq \frac{\partial u}{\partial c_{t+1}}\left(s_{0}^{\prime}\right) r_{i, t+1}+\frac{\partial u}{\partial c_{t+1}} r_{i, t+1}\left(s_{0}^{\prime}\right) \text {. }
$$

Since Choquet expectation is monotone, we have

$$
E_{t}^{C}\left[\frac{\partial u}{\partial c_{t+1}} r_{i, t+1}+\frac{\partial u}{\partial c_{t+1}}\left(s_{0}^{\prime}\right) r_{i, t+1}\left(s_{0}^{\prime}\right)\right] \geq E_{t}^{C}\left[\frac{\partial u}{\partial c_{t+1}}\left(s_{0}^{\prime}\right) r_{i, t+1}+\frac{\partial u}{\partial c_{t+1}} r_{i, t+1}\left(s_{0}^{\prime}\right)\right] \text {. }
$$

Given $s_{0}{ }^{\prime}, \frac{\partial u}{\partial c_{t+1}}\left(s_{0}{ }^{\prime}\right)$, and $r_{i, t+1}\left(s_{0}{ }^{\prime}\right)$ are constants. By translatability, we have ${ }^{8}$

$$
E_{t}^{C}\left[\frac{\partial u}{\partial c_{t+1}} r_{i, t+1}\right]+\frac{\partial u}{\partial c_{t+1}}\left(s_{0}^{\prime}\right) r_{i, t+1}\left(s_{0}^{\prime}\right)\|v\| \geq E_{t}^{C}\left[\frac{\partial u}{\partial c_{t+1}}\left(s_{0}{ }^{\prime}\right) r_{i, t+1}+\frac{\partial u}{\partial c_{t+1}} r_{i, t+1}\left(s_{0}^{\prime}\right)\right]
$$

By positive homogeneity, since $\frac{\partial u}{\partial c_{t+1}}\left(s_{0}{ }^{\prime}\right)$ and $r_{i, t+1}\left(s_{0}{ }^{\prime}\right) \geq 0$, we have

$$
E_{t}^{C}\left[\frac{\partial u}{\partial c_{t+1}} r_{i, t+1}\right]+\frac{\partial u}{\partial c_{t+1}}\left(s_{0}{ }^{\prime}\right) r_{i, t+1}\left(s_{0}{ }^{\prime}\right)\|v\| \geq \frac{\partial u}{\partial c_{t+1}}\left(s_{0}{ }^{\prime}\right) E_{t}^{C}\left(r_{i, t+1}\right)+r_{i, t+1}\left(s_{0}{ }^{\prime}\right) E_{t}^{C}\left(\frac{\partial u}{\partial c_{t+1}}\right) \text {, }
$$

and this holds for any $s_{0}{ }^{\prime} \in S$. That is,

$$
E_{t}^{C}\left[\frac{\partial u}{\partial c_{t+1}} r_{i, t+1}\right]+\frac{\partial u}{\partial c_{t+1}} r_{i, t+1}\|v\| \geq \frac{\partial u}{\partial c_{t+1}} E_{t}^{C}\left(r_{i, t+1}\right)+r_{i, t+1} E_{t}^{C}\left(\frac{\partial u}{\partial c_{t+1}}\right)
$$

\footnotetext{
${ }^{8}$ Choquet integrals are translatable for any real number $\beta$, such that $E_{t}^{C}(X+\beta)=E_{t}^{C}(X)+\beta\|v\|$, if $v$ is a monotone measure on the measurable space $(S, \mathcal{F})$.
} 
When both $E_{t}^{C}\left(r_{i, t+1}\right)$ and $E_{t}^{C}\left(\frac{\partial u}{\partial c_{t+1}}\right)$ are finite, apply translatability again to acquire

$$
E_{t}^{C}\left[\frac{\partial u}{\partial c_{t+1}} r_{i, t+1}\right]\|v\|+E_{t}^{C}\left[\frac{\partial u}{\partial c_{t+1}} r_{i, t+1}\right]\|v\| \geq E_{t}^{C}\left(\frac{\partial u}{\partial c_{t+1}}\right) E_{t}^{C}\left(r_{i, t+1}\right)+E_{t}^{C}\left(r_{i, t+1}\right) E_{t}^{C}\left(\frac{\partial u}{\partial c_{t+1}}\right) .
$$

Dividing the norm on both sides, we find

$$
E_{t}^{C}\left[\frac{\partial u}{\partial c_{t+1}} r_{i, t+1}\right] \geq \frac{1}{\|v\|} E_{t}^{C}\left(\frac{\partial u}{\partial c_{t+1}}\right) E_{t}^{C}\left(r_{i, t+1}\right)
$$

Since $v$ is a probability measure, $\|v\|=1$. This proves part 1 of the theorem.

If $v$ is submodular and $\frac{\partial u}{\partial c_{t+1}}$ and $r_{i, t+1}$ are countermonotonic, the second part of the theorem follows from the same logic.

\section{Proof of Lemma 2:}

Suppose $u$ is weakly increasing in $s \in S$. Given a monotone measure space, $(S, F, V)$, we denote $\left\{u_{s} \geq t\right\}=\{s \mid u(s) \geq t\}$ for any $t>0$. The Choquet integral of $u$ over $S$ with respect to a real monotone measure $v$ is

$$
\begin{aligned}
\int u_{s} v(d s) & =\int_{0}^{+\infty} v\left(\left\{i \mid u_{i} \geq t\right\}\right) d t \\
& =\int_{0}^{u_{1}} v\left(\left\{i \mid u_{i} \geq t\right\}\right) d t+\sum_{s=2}^{n} \int_{u_{s-1}}^{u_{s}} v\left(\left\{i \mid u_{i} \geq t\right\}\right) d t+\int_{u_{n}}^{+\infty} v\left(\left\{i \mid u_{i} \geq t\right\}\right) d t \\
& =\int_{0}^{u_{1}} v(\{1,2, \cdots, n\}) d t+\sum_{s=2}^{n} \int_{u_{s-1}}^{u_{s}} v(\{s, s+1, \cdots, n\}) d t+\int_{u_{n}}^{+\infty} v(\phi) d t \\
& =u_{1} f\left(\sum_{i} P_{i}\right)+\sum_{s=2}^{n}\left(u_{s}-u_{s-1}\right) f\left(\sum_{i \geq s} P_{i}\right) \\
& =\sum_{s=1}^{n} u_{s} f\left(\sum_{i \geq s} P_{i}\right)-\sum_{s=2}^{n} u_{s-1} f\left(\sum_{i \geq s} P_{i}\right) \\
& =u_{n} f\left(P_{n}\right)+\sum_{s<n} u_{s} f\left(\sum_{i \geq s} P_{i}\right)-\sum_{s<n} u_{s} f\left(\sum_{i>s} P_{i}\right) \\
& =\sum_{s=1}^{n} u_{s} P_{s}^{\uparrow} .
\end{aligned}
$$

The weakly decreasing case of $u$ can be proven likewise. 
Proof of Lemma 3: Now we prove the fact that $-E_{t}^{C}\left[-Q_{t+1} r_{i, t+1}\right]>E_{t}^{C}\left[Q_{t+1} r_{i, t+1}\right]$.

Denoting by $A(t)=\left\{s^{\prime} \in S \mid Q_{t+1}\left(s^{\prime}\right) r_{i, t+1}\left(s^{\prime}\right) \geq t\right\}$, we find

$$
E_{t}^{C}\left[Q_{t+1}\left(s^{\prime}\right) r_{i, t+1}\left(s^{\prime}\right)\right]=\int_{-\infty}^{0}[v(A(t))-1] d t+\int_{0}^{+\infty} v(A(t)) d t
$$

Based on the definition $A(t)$, consider the event $-Q_{t+1}\left(s^{\prime}\right) r_{i, t+1}\left(s^{\prime}\right)>t$ so that

$$
\begin{aligned}
& \left\{s^{\prime} \in S \mid-Q_{t+1}\left(s^{\prime}\right) r_{i, t+1}\left(s^{\prime}\right)>t\right\} \\
& =\left\{s^{\prime} \in S \mid Q_{t+1}\left(s^{\prime}\right) r_{i, t+1}\left(s^{\prime}\right)<-t\right\} \\
& =\Omega \backslash A(-t) \\
& =A(-t)^{c} .
\end{aligned}
$$

Here the superscript lower case c means complement of $A(-t)$. It should not be confused with the upper case $C$ superscipt notation for Choquet. We have therefore

$$
\begin{aligned}
E_{t}^{C}\left[-Q_{t+1}\left(s^{\prime}\right) r_{i, t+1}\left(s^{\prime}\right)\right] & =\int_{-\infty}^{0}\left[v\left(A(-t)^{c}\right)-1\right] d t+\int_{0}^{+\infty} v\left(A(-t)^{c}\right) d t \\
& =-\int_{\infty}^{0}\left[v\left(A(z)^{c}\right)-1\right] d z-\int_{0}^{-\infty} v\left(A(z)^{c}\right) d z \\
& =\int_{-\infty}^{0} v\left(A(t)^{c}\right) d t+\int_{0}^{+\infty}\left[v\left(A(t)^{c}\right)-1\right] d t .
\end{aligned}
$$

Furthermore, $E_{t}^{C}\left[Q_{t+1}\left(s^{\prime}\right) r_{i, t+1}\left(s^{\prime}\right)\right]+E_{t}^{C}\left[-Q_{t+1}\left(s^{\prime}\right) r_{i, t+1}\left(s^{\prime}\right)\right]$ yields

$$
\begin{aligned}
& E_{t}^{C}\left[Q_{t+1}\left(s^{\prime}\right) r_{i, t+1}\left(s^{\prime}\right)\right]+E_{t}^{C}\left[-Q_{t+1}\left(s^{\prime}\right) r_{i, t+1}\left(s^{\prime}\right)\right] \\
& =\int_{-\infty}^{+\infty}\left[v(A(t))+v\left(A(t)^{c}\right)-1\right] d t,
\end{aligned}
$$

by the fact that the probability is nonadditive. When the agent is uncertainty averse, $v(A)+v\left(A^{c}\right)<1$, so that $\int_{-\infty}^{+\infty}\left[v(A(t))+v\left(A(t)^{c}\right)-1\right] d t<0$. The conclusion of the lemma follows.

\section{References}

Allais, M. (1953). "Le Comportement de I'Homme Rationnel devant le Risque: Critique des Postulats et Axiomes de l'Ecole Americaine," Econometrica 21(4): 503-546. 
Barnett, W. A. (1978). "The User Cost of Money," Economics Letters 1: 145-149.

Barnett, W. A. (1980). "Economic Monetary Aggregates: An Application of Index Number and Aggregation Theory," Journal of Econometrics 14: 11-48.

Barnett, W. A. (1995). "Exact Aggregation under Risk." In W. A. Barnett, M. Salles, H. Moulin, and N. Schofield (eds.), Social Choice, Welfare and Ethics, Proceedings of the Eighth International Symposium in Economic Theory and Econometrics, Cambridge University Press, 353-374. Reprinted in W. A. Barnett and A. Serletis (2000) (eds.), The Theory of Monetary Aggregation, Amsterdam: North-Holland, Ch. 10: 195-206.

Barnett, W. A. (2012). Getting It Wrong: How Faulty Monetary Statistics Undermine the Fed, the Financial System, and the Economy, MIT Press, Cambridge, MA.

Barnett, W.A. and M. Chauvet (2011). "How Better Monetary Statistics Could Have Signaled the Financial Crisis," Journal of Econometrics 161(1): 6-23.

Barnett, W. A., J. Keating, and L. Kelly (2011). "Rethinking the Liquidity Puzzle: Applications of a New Measure of Economic Money Stock," Journal of Banking and Finance 35(4): 765-1026.

Barnett, W. A., Y. Liu, and M. Jensen (1997). "CAPM Risk Adjustment for Exact Aggregation over Financial Assets," Macroeconomic Dynamics 1: 485-512.

Barnett, W. A., and S. Wu (2005). "On User Costs of Risky Monetary Assets," Annals of Finance 1: 35-50.

Belongia, M. T. and J. A. Chalfant (1989). "The Changing Empirical Definition of Money: Some Estimates from a Model of the Demand for Money Substitutes," Journal of Political Economy 97: 387-397.

Belongia, M.T. and P.N. Ireland (2014). "The Barnett Critique after Three Decades: A New Keynesian Analysis." Journal of Econometrics 183(1): 5-21.

Belongia, M. T. and P. N. Ireland (2015a), "Interest Rates and Money in the Measurement of Monetary Policy," Journal of Business and Economic Statistics 332: $255-269$.

Belongia, M. T. and P. N. Ireland (2015b), “A 'Working' Solution to the Question of Nominal GDP Targeting, Macroeconomic Dynamics 13(3): 508-534. 
Belongia, M.T. and P.N. Ireland (2016). "Money and Output: Friedman and Schwartz Revisited." Journal of Money, Credit and Banking 48(6): 1223-1266.

Bewley. T. (2002). "Knightian Decision Theory: Part 1," Decisions in Economics and Finance, 25: 79-110.

Chateauneuf, A. and M. D. Cohen (2010). "Cardinal Extensions of the EU Model Based on the Choquet Integral." In Bouyssou, Dennis; Dubois, Didier; Mirlot, Marc; and Henri Prade, Decision-making Process: Concepts and Methods.

Choquet, G. (1954). "Theory of Capacities," Annales de l'institut Fourier 5: 131-295.

Ellsberg, D. (1961). "Risk, Ambiguity and the Savage Axioms," Quarterly Journal of Economics 75: 643-669.

Epstein, L. G., and S. E. Zin (1989). "Substitution, Risk Aversion, and the Temporal Behavior of Consumption and Asset Returns: A Theoretical Framework," Econometrica 57(4): 937-969.

Gilboa, I. (1987). "Expected Utility Theory with Purely Subjective Non-Additive Probabilities," Journal of Mathematical Economics 16: 65-88.

Gilboa, I. (2009). Theory of Decision under Uncertainty. New York: Cambridge University Press.

Gilboa, I., and D. Schmeidler (1989). "Maxmin Expected Utility with a Non-Unique Prior," Journal of Mathematical Economics 18: 141-153.

Grabisch, J. (1996). "The Application of Fuzzy Integrals in Multicriteria Decision Making," European Journal of Operational Research 89: 445-456.

Jadidzadeh, Ali and A. Serletis (2018), "The Demand for Assets and Optimal Monetary Aggregation," Journal of Money, Credit and Banking, forthcoming.

Kahneman, D., and A. Tversky (1979). "Prospect Theory: An Analysis of Decision under Risk," Econometrica 47(2): 263-292.

Keating, J., and A. Lee Smith (2018). "Are Monetary Aggregates Useful in Taylor Rules? Evidence from a DSGE Model with a Financial Sector," University of Kansas, working paper.

Knight, F. H. (1921). Risk, Uncertainty, and Profit, Boston/New York: Houghton Mifflin. Poterba, J.M. and J.J. Rotemberg (1987). “Money in the Utility Function: An Empirical 
Implementation," Chapter 10 in W.A. Barnett and K.J. Singleton (eds), New Approaches to Monetary Economics. Cambridge: Cambridge University Press: 219-40.

Savage, L. J. (1954). The Foundations of Statistics, New York: John Wiley and Sons. (Second Edition, 1972, New York: Dover).

Schmeidler, D. (1986). "Integral Representation without Additivity," Proceedings of the American Mathematical Society 97: 255-261.

Schmeidler, D. (1989). "Subjective Probability and Expected Utility without Additivity," Econometrica 57: 571-587.

Serletis, A. and P. Gogas (2014). "Divisia Monetary Aggregates, the Great Ratios, and Classical Money Demand Functions," Journal of Money, Credit and Banking 46(1): $229-41$

Serletis, A. and S. Rahman (2013). "The Case for Divisia Money Targeting," Macroeconomic Dynamics 17: 1638-1658.

Thaler, Richard (1993). Advances in Behavioral Finance, Russell Sage Foundation, New York, NY.

Tversky, A. and D. Kahneman (1992). “Advances in Prospect Theory: Cumulataive Representation of Uncertainty," Journal of Risk and Uncertainty 5: 297-323.

Weil, P. (1990). "Nonexpected Utility in Macroeconomics," Quarterly Journal of Economics 105: 29-42.

Yaari, M. E. (1987). "The Dual Theory of Choice under Risk," Econometrica 55(1): $95-115$. 